Research Article

Cellular, Molecular and Developmental Genetics

\title{
Combining canine mesenchymal stromal cells and hyaluronic acid for cartilage repair
}

\author{
Maria Inês Wits ${ }^{1}$, Gabriela Cabanas Tobin ${ }^{2}$, Maiele Dornelles Silveira ${ }^{2,3}$, Karine Gehlen Baja ${ }^{1}$, Luisa Maria \\ Macedo Braga $^{3}$, Patricia Sesterheim ${ }^{4}$, Melissa Camassola ${ }^{2}$ and Nance Beyer Nardi ${ }^{2,3,4}$ (D) \\ ${ }^{1}$ Universidade Luterana do Brasil, Hospital Veterinário, Canoas, RS, Brazil. \\ ${ }^{2}$ Universidade Luterana do Brasil, Laboratório de Células-Tronco e Engenharia de Tecidos, Canoas, RS, \\ Brazil. \\ ${ }^{3}$ CellMed Medicina Regenerativa, Porto Alegre, RS, Brazil. \\ ${ }^{4}$ Fundação Universitária de Cardiologia, Instituto de Cardiologia do Rio Grande do Sul, Porto Alegre, RS, \\ Brazil.
}

\begin{abstract}
Cell therapy and tissue engineering have been intensively researched for repair of articular cartilage. In this study, we investigated the chondrogenic potential of canine adipose-derived mesenchymal stromal cells (ASCs) combined to high molecular weight hyaluronic acid (HA) in vitro, and their therapeutic effect in dogs with chronic osteoarthritis (OA) associated with bilateral hip dysplasia. Canine ASCs were characterized after conventional 2D culture or 3D culture in $\mathrm{HA}$, showing adequate immunophenotype, proliferation and trilineage differentiation, as well as chondrogenesis after cultivation in HA. ASC/HA constructs were used to treat 12 dogs with $\mathrm{OA}$, sequentially assigned to control, ASC and ASC/HA groups. Animals were examined for clinical, orthopedic and radiological parameters. Lameness at walk and pain on manipulation were reduced in the ASC group and mainly in the ASC/HA group. Range of motion and detection of crepitus on hip rotation and abduction improved similarly in all groups. For articular edema, muscle atrophy, Norberg angle values and radiographic analyses, there were no variations throughout the period. These results indicate that ASC/HA constructs are safe and may be an effective therapeutic tool in treating canine chronic osteoarthritis, which should be confirmed with larger studies and additional clinical parameters.
\end{abstract}

Keywords: Mesenchymal stromal cells, hyaluronic acid, osteoarthritis, dog.

Received: August 17, 2019; Accepted: January 23, 2020.

\section{Introduction}

Osteoarthritis $(\mathrm{OA})$ is a degenerative disease characterized by the progressive deterioration of articular cartilage and structural changes to the entire synovial joint. Also referred to as degenerative joint disease or osteoarthrosis, $\mathrm{OA}$ is one of the most prevalent causes of severe pain and chronic physical disability in humans and dogs (Vina and Kwoh, 2018). Since the articular cartilage is hypocellular, avascular and aneural, the turnover of extracellular matrix (ECM) molecules is very slow, which limits its repair capacity (Varela-Eirin et al., 2018). Current palliative treatments, including combinations of non-steroidal antiinflammatory drugs, analgesics, nutraceuticals, functional foods, physiotherapy and alternative therapies such as acupuncture, often do not provide complete pain relief. Nonpharmaceutical treatment options may also include sur-

Send correspondence to Nance Beyer Nardi. Fundação Universitária de Cardiologia, Instituto de Cardiologia do Rio Grande do Sul, Av Princesa Isabel 370, 90040-371 Porto Alegre, RS, Brazil. E-mail: nancenardi@gmail.com. gery, weight loss, exercise modification, and physical therapy. Cell therapy and tissue engineering have emerged as potential candidates, using cells such as mesenchymal stromal cells or MSCs (Szychlinska et al., 2017).

MSCs exist in virtually all tissues and play a significant role in tissue repair and regeneration (da Silva Meirelles et al., 2006, 2008). Their high therapeutic potential is mainly due to their capacity to be activated by signals secreted by injured tissues and production of bioactive molecules (da Silva Meirelles et al., 2016). A large number of studies on the biological properties of MSCs have shown their potential for the treatment of OA in humans, by increasing chondrocyte proliferation rates and extracellular matrix production, as well as by their immunomodulatory capacity (Kong et al., 2017). Results from preclinical and clinical studies show that intra-articular injection of MSCs can reduce pain and improve the joint's function, but also suggest that more studies are required for higher levels of evidence (reviewed in Sasaki et al., 2019; Wang et al., 2019). 
The association of MSCs with three-dimensional (3D) scaffolds can increase their therapeutic potential (reviewed in Szychlinska et al., 2019). Many types of biomaterials have been used, in the search for molecules with biological activity. Among them, hyaluronic acid (HA), a large glycosaminoglycan present in the synovial fluid and with reduced concentration in patients with knee OA $(\mathrm{Ba}-$ lazs et al., 1967; Altman, 2003), has been investigated. High molecular weight hyaluronic preparation hylan GF20 , a commercially available hydrogel, is a cross-linked preparation of hyaluronan with a molecular weight of 6,000 $\mathrm{kDa}$ (Pavelka and Uebelhart, 2011) which has been successfully used as viscosupplementation for knee OA in humans and dogs (Pavelka and Uebelhart, 2011; Shen et al., 2014; Carapeba et al., 2016). However, the effect of viscosupplementation is limited, so that it could be increased and particularly extended for longer periods by the combination with MSCs.

The association of MSCs with HA to treat cartilage has been studied in different species, mostly animal models. Although the treatments are safe and promising results are observed, it is still not possible to draw conclusions on their real benefits, due to the limitations inherent to artificial models and to the heterogeneity and low quality of many of the clinical studies (Roffi et al., 2018). It has been stressed that the use of naturally occurring diseases in companion animals can provide more reliable information in translational medicine (Kol et al., 2015; Hoffman and Dow, 2016). The present work aimed at determining the potential of high molecular weight hyaluronic acid to support the chondrogenic differentiation of canine ASCs in vitro, as well as the therapeutic potential of ASCs in combination with HA in dogs suffering from osteoarthritis.

\section{Materials and Methods}

\section{Animals}

Four healthy male dogs submitted to surgical intervention for procedures of castration were used as donors of subcutaneous adipose tissue samples, with prior signing of informed consent by the owners. Twelve dogs of different breeds with bilateral OA of the hip joint were recruited for treatment, as detailed below. The protocol was approved by the Animal Ethics Committee of Universidade Luterana do Brasil (no. 2016.82).

\section{Reagents, culture media and solutions}

Complete culture medium consisted of Dulbecco's modified Eagle's Medium (DMEM) supplemented with HEPES (free acid, 2.5-3.7 g/l) and 10\% fetal bovine serum (Life Technologies do Brasil, SP, Brazil). Hank's balanced salt solution containing $10 \mathrm{mM}$ sodium HEPES (HBSS) was used for washing and resuspending the cells. All reagents used in this study were from Sigma-Aldrich Brasil
Ltda (SP, Brazil), unless otherwise stated. Plasticware was from Greiner Bio-One Brasil (SP, Brazil).

\section{Isolation, culture and characterization of canine ASCs}

Subcutaneous adipose tissue was digested with collagenase type I, and cultured at $37{ }^{\circ} \mathrm{C}$ as previously described (da Silva Meirelles et al., 2006, 2016). Cells between passages 3 and 5 were used for all experiments and for treating OA dogs, as described below. Experiments were done in triplicate.

ASCs were analyzed for morphology, surface phenotype, proliferation and differentiation potential. Cultures were observed under an inverted phase-contrast microscope (Axiovert 25, Zeiss, Hallbergmoos, Germany). Photomicrographs were taken with a digital camera (AxioCam MRc, Zeiss), using the AxioVision 3.1 software (Zeiss). Cell proliferation rate was assessed by counting the number of cells recovered in each passage, as well as the time elapsed. The results are expressed as the number of cells over the days of culture.

The trilineage differentiation potential of ASC cultures was analyzed by incubation for up to 4 weeks with differentiation-inducing culture media (da Silva Meirelles et al., 2016). Adipocytes, osteoblasts, and chondrocytes were revealed with specific staining solutions (Oil Red O, Alizarin Red S, and Alcian Blue, respectively). All procedures had negative control cultures (undifferentiated cultures).

For immunophenotyping, cultures derived from one donor were analyzed in triplicate. The cells were incubated for $30 \mathrm{~min}$ at room temperature with antibodies specific for canine CD45, MHC class II or CD90 and conjugated with fluorescein isothiocyanate (FITC) or R-phycoerythrin (PE) (eBioscience, La Jolla, CA, USA). The cells were analyzed on an ACCURI C6 flow cytometer (Becton Dickinson, USA). At least 10,000 events were collected, and the results were analyzed with the BD Accuri C6 software.

\section{Proliferation and differentiation of ASCs in HA constructs}

For encapsulation in high molecular weight hyaluronic acid (Synvisc $\AA$, Sanofi Brasil, Brazil), ASCs were resuspended in DMEM at $8 \times 10^{5}$ cells $/ \mathrm{mL}$, mixed with HA at $1: 10(\mathrm{v}: \mathrm{v})$ and dispensed into $24-$ well plates $(0.5 \mathrm{ml} /$ well). After $3 \mathrm{~h}$ incubation at $37^{\circ} \mathrm{C}$ for cell attachment, the scaffolds were transferred to 12 -well plates and submerged in control or chondrogenic-inducing medium. The cell/HA constructs were maintained at $37^{\circ} \mathrm{C}$ with $5 \% \mathrm{CO}_{2}$ for up to 28 days, with medium change every 3 or 4 days.

After 3 days in culture, the proliferation of ASCs encapsulated in HA was determined with a Quant-iT PicoGreen dsDNA kit (Molecular Probes, Invitrogen), which allows quantitating double-stranded DNA. Measurements were performed using a SpectraMax (Molecular Devices, 
Sunnyvale, CA, USA) plate reader at $485 \mathrm{~nm}$ excitation and $535 \mathrm{~nm}$ emission wavelengths.

Cultures were photographed as described above on days 7, 21 and 28 for analysis of morphology. On day 28, the constructs were stained with $0.5 \%$ Alcian blue $(\mathrm{pH} 2.5)$, to detect production of cartilaginous matrix proteoglycans. Samples collected for histological analysis were fixed in $4 \%$ paraformaldehyde and embedded in paraffin blocks. Sections of $4-\mu \mathrm{m}$ thickness were mounted on glass slides and stained with Alcian blue. All sections were evaluated under light microscope. Non-seeded HA scaffolds were also stained, as controls. ASCs from three donors were analyzed, and experiments were done in triplicate.

Sulphated glycosaminoglycans (GAGs) were measured using the dimethyl blue (DMB) technique. ASC-encapsulated constructs were collected on day 28 and digested overnight in phosphate buffer $50 \mathrm{mM} \mathrm{pH} 6.5$ with $0.24 \mathrm{~g} / \mathrm{L}$ L-cysteine and $0.4 \%$ EDTA $0.5 \mathrm{M}$. After mixing with chloroform and centrifuged at 10,000 $\mathrm{x} g$ for $15 \mathrm{~min}$ utes, $4{ }^{\circ} \mathrm{C}, 25 \mu \mathrm{L}$ of the supernatant was mixed with freshly prepared DMB solution (DMB $0.3 \mathrm{~mol} / \mathrm{L}$ with $2 \mathrm{~mol} / \mathrm{L}$ Tris) and absorbance was read at $530 \mathrm{~nm}$ in a plate reader (SpectraMax M3, Molecular Devices). Protein content was determined using the Lowry method.

\section{Treatment of dogs with chronic osteoarthritis}

To investigate the therapeutic potential of ASCs and ASC/HA constructs in osteoarthritis, ASCs alone or encapsulated in hyaluronic acid were used to treat OA-affected dogs. Twelve adult client-owned dogs were included in the study. Inclusion criteria were age ( $\leq 6$ years old), lameness and pain due to OA associated with bilateral hip dysplasia. Exclusion criteria were previous surgery, any kind of treatment during the previous two months, dislocation of the affected joint and presence of infections, cancer or other diseases which could interfere with interpretation of the results.

The animals were sequentially assigned to three groups ( $n=4$ /group). ASCs and ASC/hyaluronic acid constructs, prepared as described above, were used for all dogs. The control group received a single intra-articular injection of $0.5 \mathrm{~mL}$ phosphate buffered saline (PBS) per joint. The ASC group received $5 \times 10^{6}$ cells in $0.5 \mathrm{~mL}$ PBS per joint, and the ASC/HA group was treated with $5 \times 10^{6}$ ASCs encapsulated in $0.5 \mathrm{~mL}$ hyaluronic acid per joint. Dogs were sedated and injected into single, aseptically prepared sites on both hip joints.

On the first evaluation (day zero), the dogs were examined for clinical, orthopedic and radiological parameters. Clinical and orthopedic results were independently evaluated by two veterinarians, blinded to treatment allocation, on days 7, 30, 60 and 90 after treatment, and another radiographic examination was performed on day 90 . The clinical and orthopedic examination used a numerical rating scale, in which lameness, pain on manipulation, articu- lar edema, range of motion and muscle atrophy were scored from 0 to 3 (or to 4 , in the case of lameness) according to the severity of symptoms. The detection of crepitus on hip rotation and abduction was also evaluated. The score ranged from 1 (no crepitus) to 5 (crepitus with increasing degree of pain sensation).

Ventrodorsal radiographs were taken on days 0,30 , 60 and 90 and evaluated according to the evidences of instability or degenerative changes of the hip joint. Norberg angle values were measured from the hip extended view (Culp et al., 2006).

\section{Statistical analyses}

Results are expressed as mean \pm standard deviation. Data were analyzed and graphs were generated using the Prism 5 software (GraphPad Software Inc, San Diego, CA, USA). Data were tested for normality and analyzed by one-way analysis of variance (ANOVA) followed by Tukey's post hoc test. A $p$-value less than 0.05 was considered significant for all analyses.

\section{Results}

\section{Characterization of canine ASCs}

Cells had the characteristic fibroblast morphology of mesenchymal-type stem cells and showed tri-lineage differentiation potential (Figure 1A). The cells expanded rapidly for a period of 40 days, when the expansion rate decreased (Figure 1B). Cells were negative for CD45 and $\mathrm{MCH}$ class II antigens, and positive for $\mathrm{CD} 90$ (Figure 1C).

\section{Characterization of ASC/HA constructs}

The proliferation rate of ASCs cultured for 3 days in the HA, as well as control cultures maintained in the conventional 2D system, were fluorometrically assessed by the PicoGreen assay. ASCs from 3 donors were analyzed, and the assays were performed in triplicate. Cells cultured in HA had proliferation rates similar to control cultures (Figure 2).These results were also observed when the GAG contents were determined in ASC-encapsulated constructs cultured for 28 days (Figure 5). GAGs were significantly more abundant in constructs cultured with chondrogenicinducing medium.

ASC-encapsulated HA constructs were also analyzed histologically, after 28 days culture in chondrogenic-inducing medium. Sections stained with Alcian blue showed in greater detail the chondrogenic phenotype of the constructs (Figure 6).

\section{Treatment of dogs with $\mathrm{OA}$}

Twelve dogs were included in the study (Table 1). Each joint was considered as an independent sample, considering their individual characteristics, and were treated and assessed individually. The results of the evaluations by 


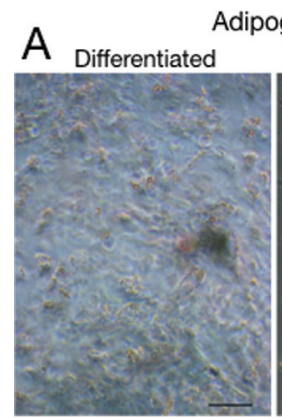

B
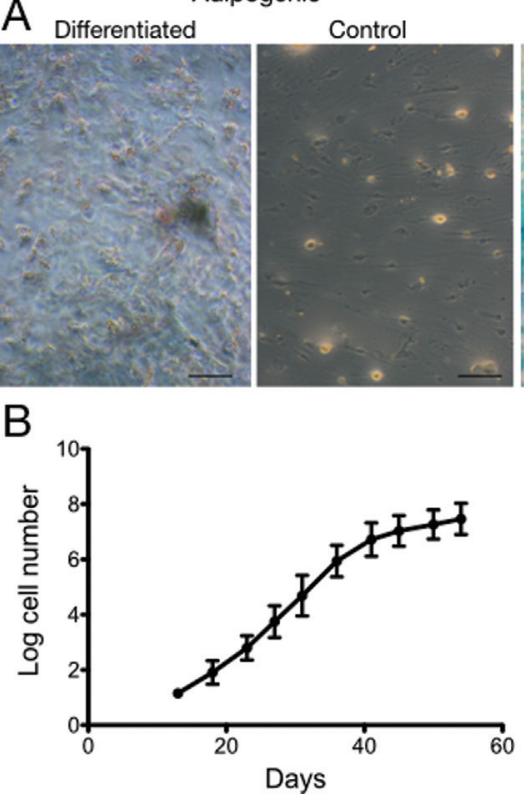

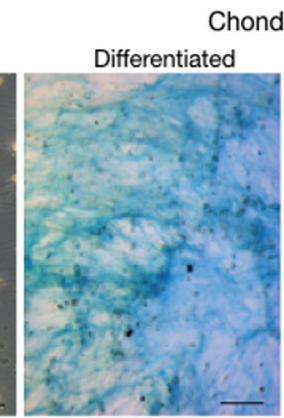

Chondrogenic

C

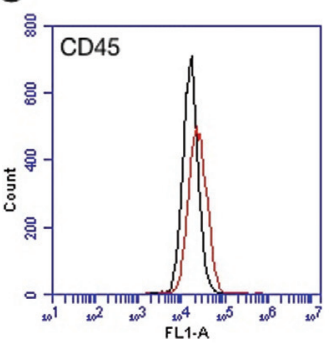

Control
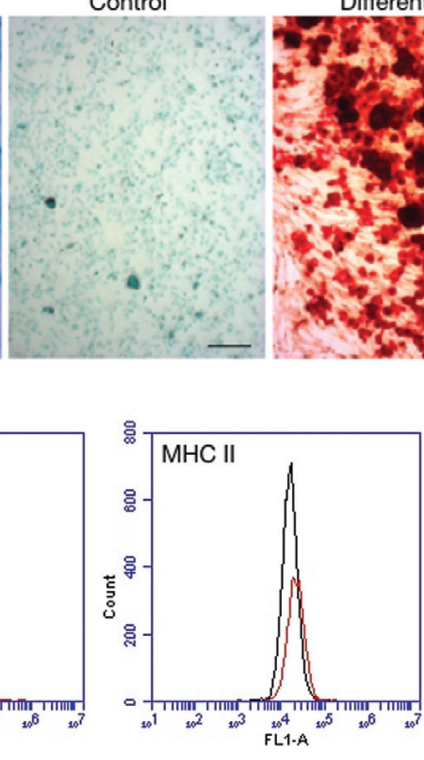

Osteogenic
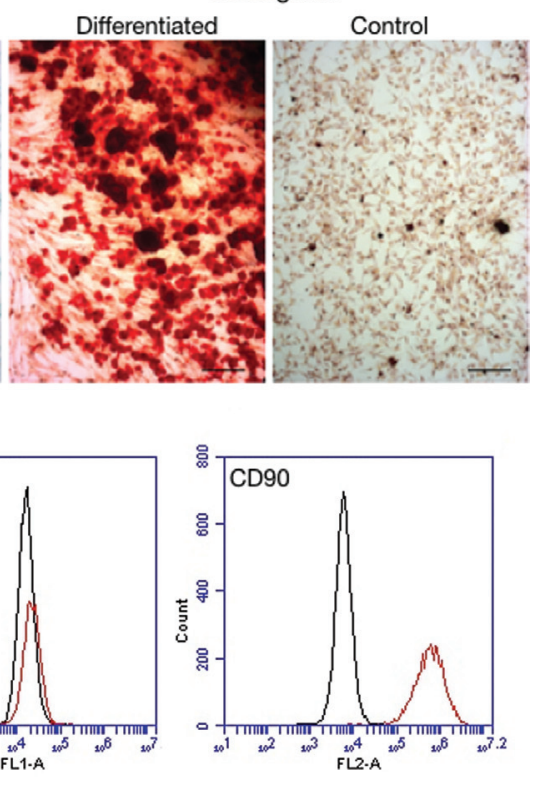

Figure 1 - (A) Representative photographs showing the trilineage differentiation capacity of canine ASCs; control cultures were maintained in normal medium. Scale bars, $100 \mu \mathrm{m}$. (B) ASC proliferation rate along 60 days of culture. (C) Immunophenotype of canine ASCs ( $\mathrm{n}=1$, analyzed in triplicate). Representative results show that cultures were negative for CD45 and MHC class II, and positive for CD90.

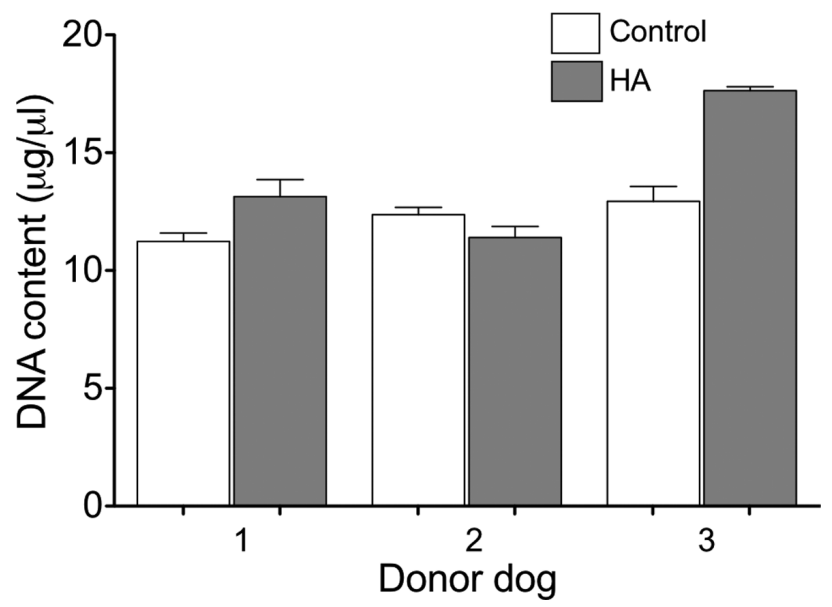

Figure 2 - Proliferation rate of canine ASCs cultured for 3 days in normal 2D conditions (Control) or encapsulated in HA, assessed by DNA assay ( $\mathrm{n}$ $=3)$.
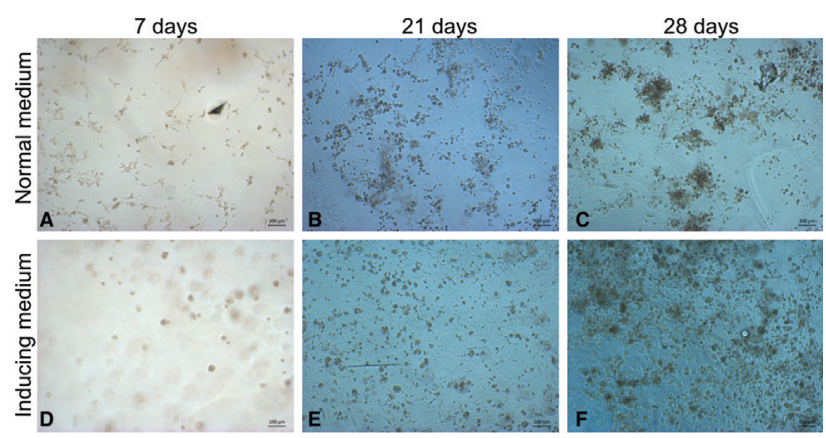

Figure 3 - Phase contrast aspect of ASCs encapsulated in HA and cultured for 7, 21 or 28 days in normal medium (A, B, C) or chondrogenic-inducing medium $(\mathrm{D}, \mathrm{E}, \mathrm{F})$. Scale bars $=100 \mu \mathrm{m}$.
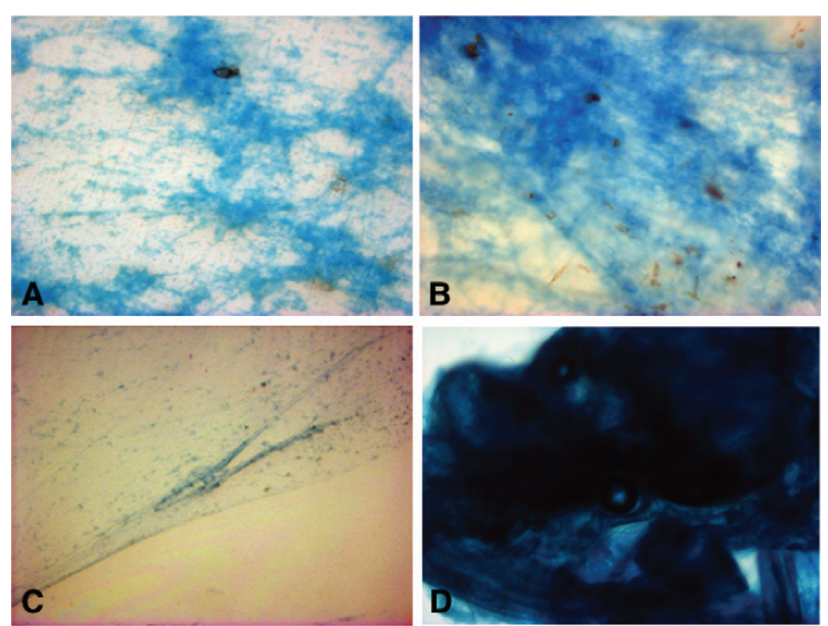

Figure 4 - Chondrogenic matrix stained with Alcian blue in encapsulated ASC/HA constructs cultured for 28 days in normal (A) or chondrogenic-inducing (B) medium. A weak background staining can be seen in HA alone (C). (D) Detail of a more dense chondrogenic structure which can also be observed in the constructs. Original magnification x200 (A and B) or $\mathrm{x} 100(\mathrm{C}$ and $\mathrm{D})$.

two veterinarians on day 0 and days 7, 30, 60 and 90 after treatment, were analyzed as described above.

No significant differences $(p>0.05)$ were observed by investigator for any outcome variable, so the data were pooled together. No adverse events related to the procedure or the treatments were observed during all the follow-up period. Lameness at walk remained unchanged in control animals, but was reduced in the ASC group $(p<0.05$ on day 90 comparing to day 0 ) and mainly in the ASC/HA group 


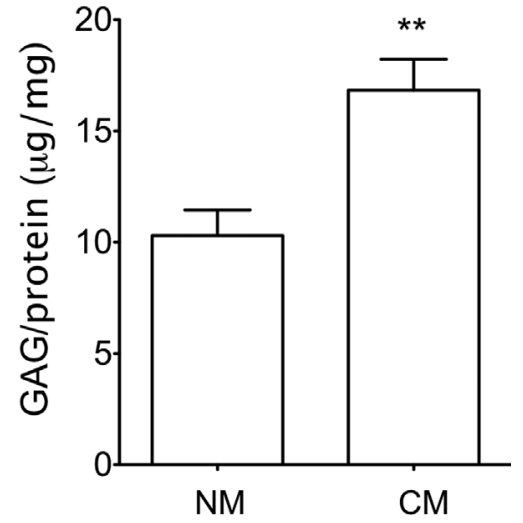

Figure 5 - GAG $(\mu \mathrm{g})$ quantification normalized to protein concentration (mg) of ASC-HA constructs cultured for 28 days $(\mathrm{n}=3) .{ }^{* *} p<0.01$.

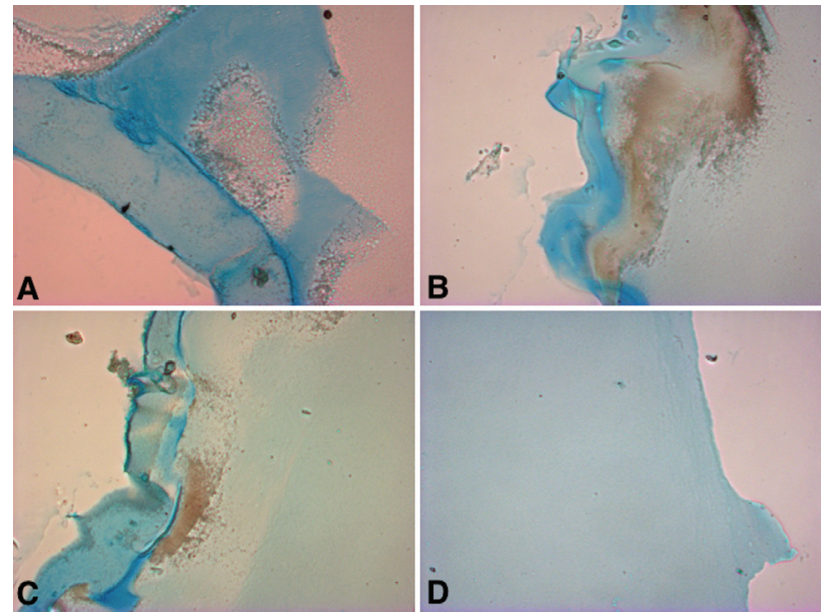

Figure 6 - Representative cross-section images of encapsulated ASC/HA constructs cultured for 28 days with chondrogenic-inducing medium, stained with Alcian blue. (A, B, C) Different aspects of the chondrogenic matrix. (D) Control (Synvisc $\AA$ alone). Original magnification x100.

Table 1 - Basic characteristics of dogs included in the study.

\begin{tabular}{lcccc}
\hline Group $^{\text {a }}$ & Breed & Sex & Age & Weight $(\mathrm{kg})$ \\
\hline Control & German shepherd & Female & 5 years & 33 \\
& Border collie & Male & 3 years & 30 \\
& Undefined & Male & 11 months & 27 \\
& Golden retriever & Female & 3 years & 30 \\
ASC & Pit bull & Female & 5 years & 26 \\
& Border collie & Female & 7 months & 13 \\
& Cimarron & Male & 1 year & 32 \\
& Rottweiler & Female & 1 year & 51 \\
ASC/HA & German shepherd & Male & 2 years & 30 \\
& Undefined & Male & 8 months & 9 \\
& Golden retriever & Male & 2 years & 36 \\
& Undefined & Male & 3 years & 53 \\
\hline
\end{tabular}

${ }^{a}$ Control: treated with PBS; ASC: treated with adipose tissue-derived mesenchymal stem cells; ASC/HA: treated with ASCs + hyaluronic acid.
( $p<0.05$ on days 60 and 90 comparing to day 0) (Figure $7 \mathrm{~A})$. A similar pattern was observed for pain on manipulation, with an earlier (30 days) and more intense effect ( $p<$ 0.01 ) in animals treated with ASCs + hyaluronic acid (Figure 7B). Range of motion and detection of crepitus on hip rotation and abduction showed continuous improvement in all groups, but without significant differences (Figure 7C and 7D). For articular edema and muscle atrophy, there were no variations throughout the period in different groups (not shown). Radiographic analyses (not shown), as well as Norberg angle measurements (Table 2), did not show any differences when pretreatment results were compared to results on days 30,60 and 90 .

\section{Discussion}

Mesenchymal stem cells have attracted much attention for their potential in tissue engineering, mainly due to their plasticity and secretion of paracrine factors with regenerative functions ( $\mathrm{Im}, 2018)$. The cells isolated from canine adipose tissue and cultured in the present study were analyzed for morphology, surface profile, proliferation and trilineage differentiation, with results that characterize them as cells of the mesenchymal lineage (Konno et al., 2013). Of particular importance for the treatment of osteoarthritis, canine ASCs differentiated readily into the chondrogenic lineage, similar to previously published results (Marx et al., 2014).

The behaviour of ASCs encapsulated in high molecular weight hyaluronic acid was followed during in vitro culture. The cells distributed homogeneously into the HA scaffold, in a 3D configuration. Encapsulation is the more frequently used cell seeding system in this kind of material, since their structure controls cell distribution and ECM diffusion, providing a more adequate environment for tissue engineering (Nicodemus and Bryant, 2008). The 3D cell distribution is important in determining the success of tissue-engineering products, allowing for instance the movement of cells away from the construct to promote chondral integration with the surrounding cartilage (Wu et al., 2018).

Hyaluronic acid has been used to construct hydrogels for cartilage tissue engineering in different combination. A hybrid scaffold combining HA hydrogel with porous poly(e-caprolactone) showed mechanical properties similar to that of human articular cartilage and the ability to maintain the viability, proliferation and phenotype of

Table 2 - Norberg angle measurements before treatment and on follow-up.

\begin{tabular}{lcccc}
\hline Group $^{\mathrm{a}}$ & Day 0 & Day 30 & Day 60 & Day 90 \\
\hline Control & $102.8 \pm 9.7$ & $106.0 \pm 12.2$ & $106.5 \pm 12.1$ & $105.4 \pm 14.2$ \\
ASC & $82.2 \pm 14.5$ & $82.1 \pm 13.3$ & $83.0 \pm 11.3$ & $82.6 \pm 12.3$ \\
ASC/HA & $83.4 \pm 10.0$ & $82.5 \pm 11.2$ & $83.1 \pm 12.2$ & $82.1 \pm 11.7$ \\
\hline
\end{tabular}

${ }^{a}$ Control: treated with PBS; ASC: treated with adipose tissue-derived mesenchymal stem cells; ASC/HA: treated with ASCs + hyaluronic acid. 

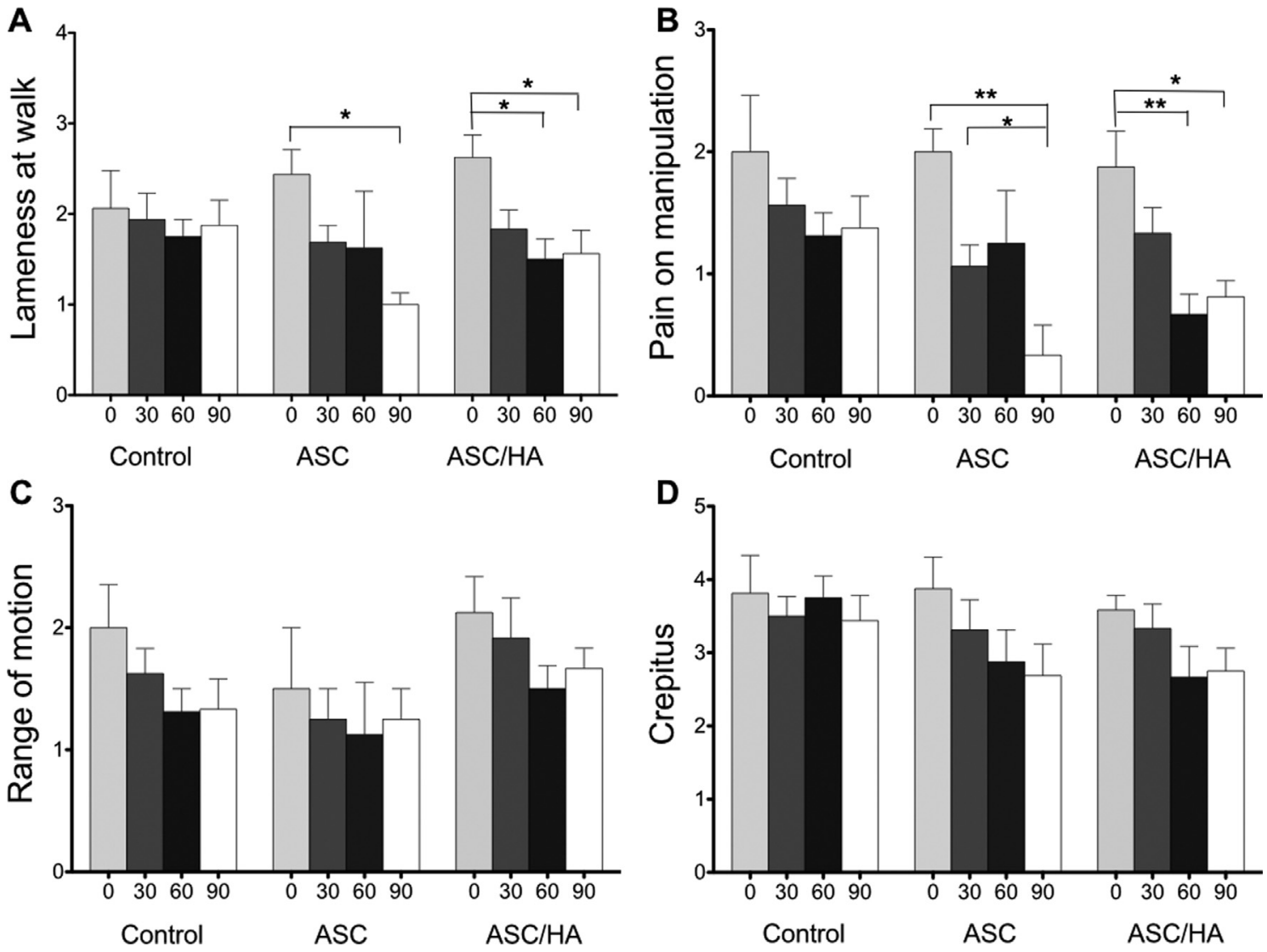

Figure 7 - Results of baseline (day 0) or follow-up (days 30, 60 and 90) evaluations of patients in groups control (treated with PBS), ASC (treated with adipose tissue-derived mesenchymal stromal cells) and ASC/HA (treated with ASCs + hyaluronic acid). ${ }^{*} \mathrm{p}<0.05 ;{ }^{* *} \mathrm{p}<0.001$, ANOVA and Tukey's post hoc test.

seeded chondrocytes (Mintz and Cooper, 2014). The proliferation, chondrogenic differentiation and therapeutic potential of mesenchymal stem/stromal cells derived from the bone marrow (de Vries-van Melle et al., 2014) or adipose tissue (Wang et al., 2018) of different species were increased by culture in HA-pNIPAM (poly(N-isopropyl acrylamide) side-chains), HA/Fibrin and HA-PNIPAAmCL (poly(N-isopropylacrylamide)) (de Vries-van Melle et al., 2014; Wang et al., 2018; Wu et al., 2018), but not in alginate/HA (de Vries-van Melle et al., 2014).

The combination ASC-HA was used for further in vivo investigation in dogs with chronic osteoarthritis. Twelve adult dogs of different breeds, with lameness and pain due to OA associated with bilateral hip dysplasia, were treated with a single intra-articular injection of PBS (control group), $5 \times 10^{6}$ allogeneic ASCs (ASC group) or $5 \times 10^{6}$ ASCs encapsulated in hyaluronic acid (ASC/HA group). No modifications in articular edema, muscle atrophy, Norberg angle or radiographic analyses were observed in the 90-day follow-up period. Interestingly, the randomization process allocated dogs with higher NA values to the control group, resulting in a slightly better evolution of this grupo, although the differences were not statistically significant.
These results reflect the need for a larger sample number in this kind of study.

Lameness at walk was improved in treated dogs, particularly in the ASC/HA group. A similar pattern was observed for pain on manipulation, with an earlier and more intense effect in ASC/HA animals.

Different animal species have been used in preclinical cell therapy studies, but there are few reports on the use of mesenchymal stem cells to treat spontaneously occurring diseases in veterinary patients. A recent review described only seven reports of cell therapy in dogs, two in cats, and four in horses, published until 2015 (Marx et al., 2015). Among the canine studies, six involved treatment of orthopedic conditions, reporting in general clinical improvement after the therapy (Marx et al., 2015). A more recent study described the treatment of 39 dogs suffering from elbow dysplasia and osteoarthritis with allogeneic ASCs, reporting highly significant clinical improvement after one year (Kriston-Pál et al., 2017). A larger study has also described beneficial effects of ASC treatment in osteoarthritis. Among 203 dogs with degenerative arthritis treated with allogeneic ASCs by intra-articular and/or intravenous injections, $88 \%$ showed improvement in clinical symptoms, whereas $12 \%$ did not exhibit any change. The response was 
significantly linked to the age of the animal, with excellent improvement in $90 \%$ of young dogs ( $<9$ years) and $60 \%$ of older dogs (Shah et al., 2018). Despite the large sample, this study had no control group and the number of injected cells, or vehicle used, are not described, limiting the significance of the results.

Although positive results have been described with the administration of cells suspended in saline or culture medium, as described above, the use of a carrier such as hydrogels or other types of biomaterials, particularly with chondroinductive properties such as hyaluronic acid, is expected to increase the efficacy of the therapy. Knee viscosupplementation by intra-articular injections of hyaluronic acid is a well established treatment to control primary OA symptoms (Bowman et al., 2018). A few studies have investigated the therapeutic potential of hyaluronic acid in canine osteoarthritis. In a double-blinded trial with 10 dogs with bilateral elbow OA, an intra-articular injection of HA supplemented with methylprednisolone was as effective as autologous conditioned plasma in improving lameness in up to 24 weeks after injection (Franklin and Cook, 2013). The efficacy of the intra-articular hyaluronic acid injection was also compared to traditional conservative treatment in 16 dogs with osteoarthritis. Clinical parameters improved in the HA-treated group from 15 to 90 and 60 to 90 days, compared to the control group (Carapeba et al., 2016).

In humans, some studies have already described the use of HA as a carrier for therapeutic cells in osteoarthritis (Park et al., 2017) and chronic discogenic low back pain (Kumar et al., 2017), or the administration of MSCs followed by hyaluronic acid in osteoarthritic patients (Lee $e t$ al., 2012; Lamo-Espinosa et al., 2018), with positive results. To our knowledge, there is only one report of a similar use of ASCs plus HA in dogs with spontaneously developed osteoarthritis. Two dogs with OA of the humeroradial joints received one intra-articular injection of autologous ASCs with Hyalgan (20 mg/2 ml; Fidia), showing functional improvements in lameness and pain on manipulation after one month (Guercio et al., 2012). The combination MSCs/HA has also been tested in dogs with a surgically induced cartilage defect, resulting in more cartilage-like tissue and significant improvement in cartilage lesions than HA alone or saline (Li et al., 2018).

The results of this study suggest a role for ASC/HA constructs in the treatment of cartilage defects, for their chondroinductive properties in vitro and effect in the treatment of canine osteoarthritis associated to hip dysplasia. These results contribute also for the development of therapeutic tools for OA in humans. Larger studies with more objective clinical evaluations, such as gait analysis and arthroscopic evaluation, are needed to validate the use of the combination of mesenchymal stromal cells and hyaluronic acid to treat chronic osteoarthritis.

\section{Acknowledgments}

This work was supported by Fundação de Amparo à Pesquisa do Estado do Rio Grande do Sul (FAPERGS); Financiadora de Estudos e Projetos (FINEP); and Conselho Nacional de Desenvolvimento Cientifico e Tecnologico (CNPq).

\section{Conflict of interest}

The authors declared no potential conflicts of interest with respect to the research, authorship, and/or publication of this article.

\section{Authors contributions}

NBN, MC and LMMB conceived and designed the study; MIW, MDS and KGB conducted the experiments; MIW, GCT, KGB, PS, MC and NBN analyzed the data; NBN and PS wrote the manuscript; all authors read and approved the final version.

\section{References}

Altman RD (2003) Status of hyaluronan supplementation therapy in osteoarthritis. Curr Rheumatol Rep 5:7-14.

Balazs EA, Watson D, Duff IF and Roseman S (1967) Hyaluronic acid in synovial fluid. I. Molecular parameters of hyaluronic acid in normal and arthritis human fluids. Arthritis Rheum 10:357-376.

Bowman S, Awad ME, Hamrick MW, Hunter M and Fulzele S (2018) Recent advances in hyaluronic acid based therapy for osteoarthritis. Clin Transl Med 7:6.

Carapeba GO, Cavaleti P, Nicácio GM, Brinholi RB, Giuffrida R and Cassu RN (2016) Intra-articular hyaluronic acid compared to traditional conservative treatment in dogs with osteoarthritis associated with hip dysplasia. Evid Based Complement Alternat Med 2016:2076921.

Culp WT, Kapatkin AS, Gregor TP, Powers MY, McKelvie PJ and Smith GK (2006) Evaluation of the Norberg angle threshold: a comparison of Norberg angle and distraction index as measures of coxofemoral degenerative joint disease susceptibility in seven breeds of dogs. Vet Surg 35:453-459.

da Silva Meirelles L, Chagastelles PC and Nardi NB (2006) Mesenchymal stem cells reside in virtually all post-natal organs and tissues. J Cell Sci 119(Pt 11):2204-2213.

da Silva Meirelles L, Caplan AI and Nardi NB (2008) In search of the in vivo identity of mesenchymal stem cells. Stem Cells 26:2287-2299.

da Silva Meirelles L, Bellagamba BC, Camassola M and Nardi NB (2016) Mesenchymal stem cells and their relationship to pericytes. Front Biosci (Landmark Ed) 21:130-156.

de Vries-van Melle ML, Tihaya MS, Kops N, Koevoet WJ, Murphy JM, Verhaar JA, Alini M, Eglin D and van Osch GJ (2014) Chondrogenic differentiation of human bone marrow-derived mesenchymal stem cells in a simulated osteochondral environment is hydrogel dependent. Eur Cell Mater 27:112-123.

Franklin SP and Cook JL (2013) Prospective trial of autologous conditioned plasma versus hyaluronan plus corticosteroid for elbow osteoarthritis in dogs. Can Vet J 54:881-884.

Guercio A, Di Marco P, Casella S, Cannella V, Russotto L, Purpari G, Di Bella S and Piccione G (2012) Production of canine 
mesenchymal tem cells from adipose tissue and their application in dogs with chronic osteoarthritis of the humeroradial joints. Cell Biol Int 36:189-194.

Hoffman AM and Dow SW (2016) Stem cell trials using companion animal disease models. Stem Cells 34:1709-1729.

Im GI (2018) Tissue engineering in osteoarthritis: current status and prospect of mesenchymal stem cell therapy. BioDrugs 32:183-192.

Kol A, Arzi B, Athanasiou KA, Farmer DL, Nolta JA, Rebhun RB, Chen X, Griffiths LG, Verstraete FJ, Murphy CJ et al. (2015) Companion animals: Translational scientist's new best friends. Sci Transl Med 7:308ps21.

Kong L, Zheng LZ, Qin L and Ho KKW (2017) Role of mesenchymal stem cells in osteoarthritis treatment. J Orthop Translat 9:89-103.

Konno M, Hamabe A, Hasegawa S, Ogawa H, Fukusumi T, Nishikawa S, Ohta K, Kano Y, Ozaki M, Noguchi Y et al. (2013) Adipose-derived mesenchymal stem cells and regenerative medicine. Dev Growth Differ 55:309-318.

Kriston-Pál É, Czibula Á, Gyuris Z, Balka G, Seregi A, Sükösd F, Süth M, Kiss-Tóth E, Haracska L, Uher F et al. (2017) Characterization and therapeutic application of canine adipose mesenchymal stem cells to treat elbow osteoarthritis. Can J Vet Res 81:73-78.

Kumar H, Ha DH, Lee EJ, Park JH, Shim JH, Ahn TK, Kim KT, Ropper AE, Sohn S, Kim CH et al. (2017) Safety and tolerability of intradiscal implantation of combined autologous adipose-derived mesenchymal stem cells and hyaluronic acid in patients with chronic discogenic low back pain: 1-year follow-up of a phase I study. Stem Cell Res Ther 8:262.

Lamo-Espinosa JM, Mora G, Blanco JF, Granero-Moltó F, NúñezCórdoba JM, López-Elío S, Andreu E, Sánchez-Guijo F, Aquerreta JD, Bondía JM et al. (2018) Intra-articular injection of two different doses of autologous bone marrow mesenchymal stem cells versus hyaluronic acid in the treatment of knee osteoarthritis: long-term follow up of a multicenter randomized controlled clinical trial (phase I/II). J Transl Med 16:213.

Lee KB, Wang VT, Chan YH and Hui JH (2012) A novel, minimally-invasive technique of cartilage repair in the human knee using arthroscopic microfracture and injections of mesenchymal stem cells and hyaluronic acid - a prospective comparative study on safety and short-term efficacy. Ann Acad Med Singapore 41:511-517.

Li L, Duan X, Fan Z, Chen L, Xing F, Xu Z, Chen Q and Xiang Z (2018) Mesenchymal stem cells in combination with hyaluronic acid for articular cartilage defects. Sci Rep 8:9900.

Marx C, Silveira MD, Selbach I, da Silva AS, Braga LM, Camassola M and Nardi NB (2014) Acupoint injection of autologous stromal vascular fraction and allogeneic adipose-derived stem cells to treat hip dysplasia in dogs. Stem Cells Int 2014:391274

Marx C, Silveira MD and Beyer Nardi N (2015) Adipose-derived stem cells in veterinary medicine: characterization and therapeutic applications. Stem Cells Dev 24:803-813.

Mintz BR and Cooper JA Jr (2014) Hybrid hyaluronic acid hydrogel/poly(E-caprolactone) scaffold provides mechanically favorable platform for cartilage tissue engineering studies. J Biomed Mater Res A 102:2918-2926.
Nicodemus GD and Bryant SJ (2008) Cell encapsulation in biodegradable hydrogels for tissue engineering applications. Tissue Eng Part B Rev 14:149-165.

Park YB, Ha CW, Lee CH, Yoon YC and Park YG (2017) Cartilage regeneration in osteoarthritic patients by a composite of allogeneic umbilical cord blood-derived mesenchymal stem cells and hyaluronate hydrogel: results from a clinical trial for safety and proof-of-concept with 7 years of extended follow-up. Stem Cells Transl Med 6:613-621.

Pavelka K and Uebelhart D (2011) Efficacy evaluation of highly purified intra-articular hyaluronic acid (Sinovial( $(\circledR)$ vs hylan G-F20 (Synvisc(®) in the treatment of symptomatic knee osteoarthritis. A double-blind, controlled, randomized, parallel-group non-inferiority study. Osteoarthritis Cartilage 19:1294-1300.

Roffi A, Nakamura N, Sanchez M, Cucchiarini M and Filardo G (2018) Injectable systems for intra-articular delivery of mesenchymal stromal cells for cartilage treatment: a systematic review of preclinical and clinical evidence. Int J Mol Sci 19:E3322.

Sasaki A, Mizuno M, Mochizuki M and Sekiya I (2019) Mesenchymal stem cells for cartilage regeneration in dogs. World $\mathrm{J}$ Stem Cells 11:254-269.

Shah K, Drury T, Roic I, Hansen P, Malin M, Boyd R, Sumer H and Ferguson R (2018) Outcome of allogeneic adult stem cell therapy in dogs suffering from osteoarthritis and other joint defects. Stem Cells Int 2018:7309201.

Shen Y, Fu Y, Wang J, Li G, Zhang X, Xu Y and Lin Y (2014) Biomaterial and mesenchymal stem cell for articular cartilage reconstruction. Curr Stem Cell Res Ther 9:254-267.

Szychlinska MA, Stoddart MJ, D'Amora U, Ambrosio L, Alini M and Musumeci G (2017) Mesenchymal stem cell-based cartilage regeneration approach and cell senescence: can we manipulate cell aging and function? Tissue Eng Part B Rev 23:529-539.

Szychlinska MA, D’Amora U, Ravalli S, Ambrosio L, Di Rosa M and Musumeci G (2019) functional biomolecule delivery systems and bioengineering in cartilage regeneration. Curr Pharm Biotechnol 20:32-46.

Varela-Eirin M, Loureiro J, Fonseca E, Corrochano S, Caeiro JR, Collado M and Mayan MD (2018) Cartilage regeneration and ageing: Targeting cellular plasticity in osteoarthritis. Ageing Res Rev 42:56-71.

Vina ER and Kwoh CK (2018) Epidemiology of osteoarthritis: literature update. Curr Opin Rheumatol 30:160-167.

Wang CZ, Eswaramoorthy R, Lin TH, Chen CH, Fu YC, Wang CK, Wu SC, Wang GJ, Chang JK and Ho ML (2018) Enhancement of chondrogenesis of adipose-derived stem cells in HA-PNIPAAm-CL hydrogel for cartilage regeneration in rabbits. Sci Rep 8:10526.

Wang AT, Feng Y, Jia HH, Zhao M and Yu H (2019) Application of mesenchymal stem cell therapy for the treatment of osteoarthritis of the knee: A concise review. World J Stem Cells 11:222-235.

Wu SC, Huang PY, Chen CH, Teong B, Chen JW, Wu CW, Chang JK and Ho ML (2018) Hyaluronan microenvironment enhances cartilage regeneration of human adipose-derived stem cells in a chondral defect model. Int $\mathrm{J}$ Biol Macromol 119:726-740.

Associate Editor: Ricardo G. Corrêa

License information: This is an open-access article distributed under the terms of the Creative Commons Attribution License (type CC-BY), which permits unrestricted use, distribution and reproduction in any medium, provided the original article is properly cited. 